\title{
Proposal of a new simplified fluid dynamic model for aerospace servovalves
}

\author{
Matteo D.L. Dalla Vedova ${ }^{1, *}$, and Pier Carlo Berri ${ }^{1}$ \\ ${ }^{1}$ Dept. of Mechanical \& Aerospace Engineering (DIMEAS), Politecnico di Torino, Turin, Italy
}

\begin{abstract}
Highly detailed computer models are required for design and development of modern flight control systems, capable of emulating with high accuracy the behaviour of on-board equipment. At the same time, different simplified models are needed, specifically intended for operations such as the optimization of preliminary design and the development of diagnostic or prognostic strategies. These simplified models are required to combine sufficient levels of accuracy and reliability with reduced computational costs, to minimize the computational burden associated with prognostic and optimization algorithms. In this work, we focus on electrohydraulic actuators, since they are critical subsystems in terms of safety and availability of the aircraft. Advanced monitoring and prognostic algorithms require new numerical models, combining an acceptable computational effort with a satisfying ability to simulate their performance and dynamics. To this purpose, this paper proposes a new simplified numerical model of the servovalve fluid-dynamic behaviour. This numerical algorithm, based on a very compact semi-empirical formulation, is intended to take into account in a simplified but sufficiently accurate way several typical effects related to the SV spool geometry and the operating conditions. To evaluate the approximations introduced by this model into a system-level simulation, it has been integrated into a dedicated numerical model simulating a simple electrohydraulic on-board actuator, and compared with a higher fidelity servovalve model.
\end{abstract}

\section{Introduction}

In this work, we consider a typical electrohydraulic servoactuator architecture. Specifically, we refer to a four-ways control valve (supply port S, return port R, control port 1, control port 2) coupled with a symmetrical linear jack, as shown in Figure 1. We aim at obtaining a computationally light model for prognostic applications [1], and focus in particular on the second stage sliding spool valve, as its highly nonlinear behaviour accounts for most computational burden of high-fidelity, CFD-based models [2-4]. In addition, it has been shown that strong linearity assumptions on the spool operation may noticeably degrade the accuracy of the whole servoactuator model in some operating conditions $[5,6]$.

* Corresponding author: matteo.dallavedova@polito.it 
As schematically represented in Figure 1, the valve spool displacement $x_{S}$ rules the opening and closing of the four passageways, each characterized by its overlaps or underlap, to connect each control port either to the supply or return port. This allows to provide the desired relationship between flow and absolute pressure for each control port $\left(P_{1}\right.$ and $\left.P_{2}\right)$, for given oil characteristics [5-8]. The corresponding differential pressure, regulated between the two control ports is $P_{12}=P_{1}-P_{2}$. In zero-flow conditions, each control port absolute pressure is close to the supply or return pressure when the corresponding passageway is completely opened. When the spool is in an intermediate position, the control port pressures have a progressive evolution between return pressure $\left(P_{R}\right)$ and supply pressure $\left(P_{S}\right)$ values, as it can be seen in the valve characteristic $P_{12}-x_{S}$ of Figure 2. Here, the $P_{12}-x_{S}$ curve is reported for different values of the jack flow $Q_{j}$; these data have been obtained with a high fidelity (HF) numerical simulation model, accounting for the actual pressure drops caused by the restricted passages of the valve, implemented in FORTRAN and Matlab-Simulink, and validated by comparison with certified numerical codes and experimental data [9-17].

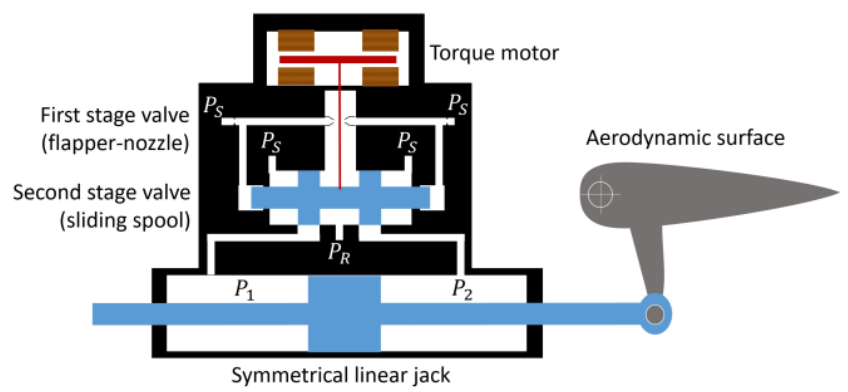

Fig. 1. Schematic cross section of a typical electrohydraulic actuator

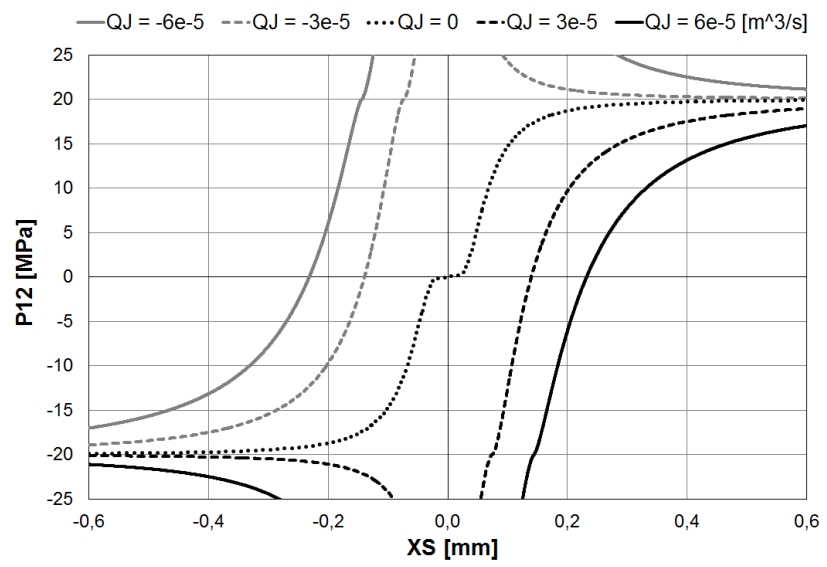

Fig. 2. Differential pressure - spool position characteristic of the considered servo-valve, as obtained by the high fidelity model

The HF model is computationally heavy and time-consuming, and it is strongly dependent on a lot of parameters related to the SV geometry and the physical characteristics of the hydraulic fluid. All those variables often are not directly available cannot be measured with sufficient accuracy. Frequently, in a simpler, lighter and quicker approach, only the controlled differential pressure between the two control ports $P_{12}$ and a single flow value $Q_{J}$ (common to both control ports) are computed with linearized models. 
Most common simplified servo-valve models available in literature simulate the fluiddynamic behaviour through a linearized approach, based on two coefficients that can be easily measured experimentally: the pressure gain $\left(G_{P}\right)$ and the flow gain $\left(G_{Q}\right)$ [7]. This results in a simplified model which can be expressed by the following equation:

$$
P_{12}=G_{P}\left(x_{S}-Q_{J} / G_{Q}\right)
$$

The spool displacement produces a proportional value of differential pressure, which acts on the motor element (i.e. the linear jack). This displacement is reduced by the pressure loss caused by the controlled flow passing through the control passageways; this effect is accounted for with the flow gain. The most evident weakness of this approach is the inability to evaluate the pressure saturation due to the limited supply value, and the actual stall conditions of the motor element.

\section{Previous simplified servovalve models}

The authors proposed in previous works [6, 18-21] several simplified models intended to modify the two gains formulation (1), in order to account for pressure saturation, leakages and water hammer. Among those, the C-type models introduce a pressure saturation downstream the Flow Gain contribution.

Model C3 was initially introduced in [21]; it takes into account the variable supply pressure and leakage acting among the control ports connecting the valve to the motor element. To account for supply pressure variation, we define $x_{S S}=P_{S R} / G_{P}$, where $P_{S R}=P_{S}$ $-P_{R}$. Despite being $P_{S R}$ and $G_{P}$ variable, $x_{S S}$ is almost constant and only depends on the valve geometry. A leakage transfer function was added downstream the pressure saturation, with a variable gain to account for and avoid interaction with the saturation itself.

A detailed explanation and derivation of C3 model is available in [21]. The resulting transfer function is:

$$
\begin{gathered}
P_{12}^{\prime}=\left\{\begin{aligned}
-P_{S R}, & x P_{S R} x_{S} / x_{S S}-G_{P Q} Q_{J}<-P_{S R} \\
x P_{S R} x_{S} / x_{S S}-G_{P Q} Q_{J}, & -P_{S R}<x P_{S R} x_{S} / x_{S S}-G_{P Q} Q_{J}<P_{S R} \\
P_{S R}, & x P_{S R} x_{S} / x_{S S}-G_{P Q} Q_{J}>P_{S R}
\end{aligned}\right. \\
P_{12}=P_{12}^{\prime} /\left(1+G_{P Q} x_{S S} C_{L k} / \max \left(\left|x_{\mathrm{S}}\right|, x_{S S}\right)\right)
\end{gathered}
$$

where $G_{P Q}=G_{P} / G_{Q}$ and $C_{L k}$ is the ratio between the leakage flow $Q_{L k}$ the control pressure $P_{12}$. The model then employs a variable gain approach, which is intended to introduce the supply pressure variation into the leakage loop, as well as the information about the actual spool position. Specifically, the pressure gain is reduced for large spool displacements, in order to account for the limited available supply pressure.

\section{Proposed model}

In this paper, the authors propose a new formulation (C5 model), derived from the previous C-type models. Model C5 is intended to overcome the problems related to the interaction between the pressure saturation block and the leakage feedback loop by modifying the formulation of the pressure/flow gain ratio $G_{P Q}$ introduced in the $\mathrm{C} 3$ model. This is achieved by introducing a new equivalent spool position $x_{\mathrm{St}}$, taking into account the effects of variable differential supply pressure $P_{S R}$ and oil flow $Q_{J}$ drained across the valve. 
Model C5 is shown by the detailed block diagram of Figure 3, and can be expressed with the following equations:

$$
\begin{aligned}
& x_{\mathrm{St}}=x_{S}-Q_{J} G_{P Q} x_{S S} / \max \left(P_{S R}, P_{\text {vap }}\right) \\
& P_{12}=x_{\mathrm{St}} P_{S R} /\left(\max \left(\left|x_{\mathrm{St}}\right|, x_{S S}\right)+C_{L k} G_{P Q} x_{S S}\right)
\end{aligned}
$$

The location of the leakage loop entirely downstream or upstream the pressure saturation block, characterizing the previous C-type models, does not seem to give a sufficiently close representation of the actual physical phenomena. In the intention of the authors, the Model C5 (schematically shown in Figure 3) should represent a development of these models capable of overcoming this drawback. It attempts to compute more efficiently and realistically the pressure gain effect in (pressure) saturation conditions by introducing a modified value of the spool displacement (called $x_{S t}$ ) which is sensitive to variations in supply pressure and oil flow. As reported in Eq. 4, the effect of the flow feedback reaction (due to the flow of oil $Q_{J}$ crossing through the regulation ports of the valve) becomes more relevant as the supply pressure is reduced (up to the corresponding vapour tension value $P_{\text {vap }}$ ). As shown in the following section, this solution simulates more realistically the cases of high spool displacement but, as can be seen from Figure 5, it is still unsatisfactory in fine regulation (i.e. for small spool openings around its centred position).

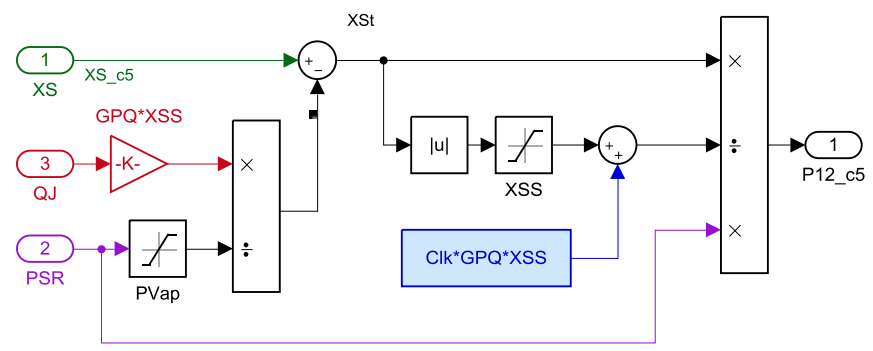

Fig. 3. Block diagram of the $\mathrm{C} 5$ model

\section{Valve fluid-dynamic characteristic}

A first evaluation of the said C-type models should be performed on the basis of the corresponding fluid-dynamic characteristics $P_{12}-x_{S}$, parameterized in $Q_{J}$. The resulting diagrams show the differential pressure $P_{12}$ acting on the motor element, for each value of $P_{S R}$ and $C_{L k}$, as a function of the displacement of the valve spool $x_{S}$, having the flow $Q_{J}$ through the piston as a parameter. The characteristics for all the considered models have been computed referring to a servo-valve featuring a value of $x_{S S}=0.1 \mathrm{~mm}, G_{P Q}=$ $6.667 \cdot 10^{11} \mathrm{~Pa} \cdot \mathrm{s} / \mathrm{m}^{3}$, and $C_{L k}=2 \cdot 10^{-13} \mathrm{~m}^{3} / \mathrm{s} / \mathrm{Pa}$, in presence of a supply pressure $P_{S R}=$ $20 \mathrm{MPa}$. The reference characteristic curve, obtained through the HF model, is shown in Figure 2, and highlights the correct simulation of water-hammer, saturation and leakage.

As regards Model C3 (Figure 4), the slope of the zero-flow curve, in its central portion, is lower than the value of $G_{P}$, since it is modified by the effect of the leakage coefficient. As shown in [21], the model is not able to take correctly into account the effect of the saturation of $P_{12}$ : in fact, for low values of spool displacement $x_{S}$, the algorithm calculates an anomalous evolution of the controlled pressure. This peculiar behaviour is due to the correction of the leakage coefficient being enabled only for $\left|x_{S}\right|>x_{S S}$, and is not representative of the real operation of the servo-valve. In particular, for $Q_{J} \neq 0$ and small $x_{S}$ (i.e. in water-hammer conditions), the experimentally detected differential pressure $P_{12}$ is usually higher (and certainly not lower) than the saturation value $P_{S R}$. 


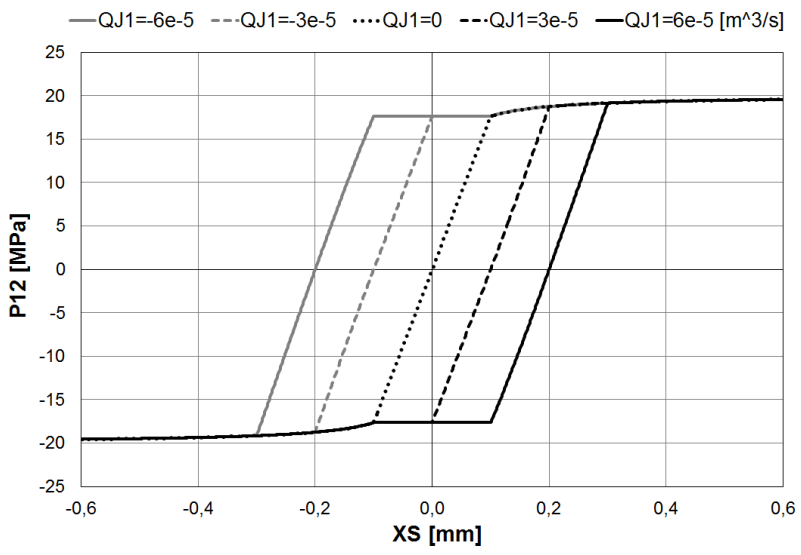

Fig. 4. Fluid-dynamic characteristic of the $\mathrm{C} 3$ model

The anomalous pressure curve of model C3 is in part improved in model C5, since the zone in which the leakage coefficient correction is enabled is modified to be sensitive to the fluid flow QJ. Figure 5 highlights how the fluid dynamic characteristic is acceptable for high spool displacements; however, C5 model still fails to correctly evaluate the water hammer effect.

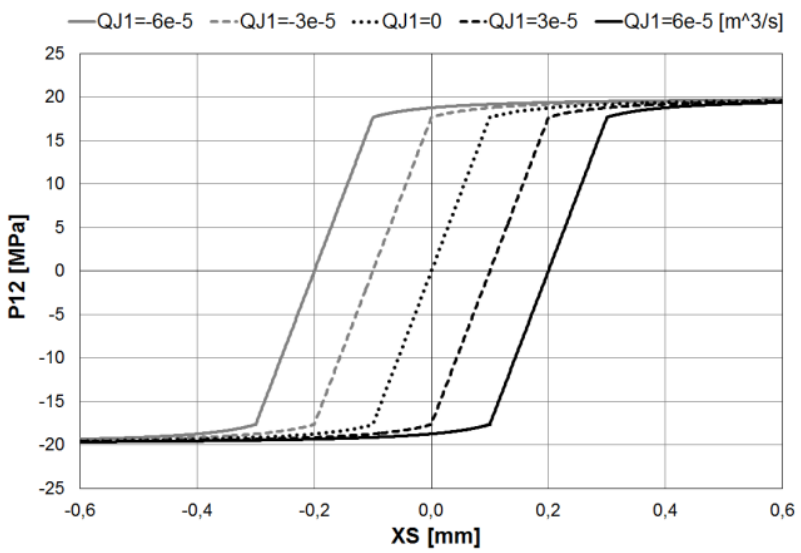

Fig. 5. Fluid-dynamic characteristic of the $\mathrm{C} 5$ model

\section{Numerical results of the EHA test bench}

The proposed simplified models are further tested by integrating them into a virtual test bench, which simulates a full position control electrohydraulic servoactuator.

In order to compare the behaviour of the different models and related computational algorithms concerning the fluid dynamics of the control valve equipping a hydraulic actuation servomechanism, a typical system was considered. Referring to the block diagram of Figure 6, it mainly consists of a Power Control and Drive Unit (PCDU) and its control is performed by an Electronic Control Unit (ECU), closing the position control loop, calculating the instantaneous position error, and generating the reference signal $i_{c}$ by means of the controller. The PCDU contains the electro-hydraulic two-stage control servo-valve and a hydraulic piston. 
The actuator HF model takes into account the main electrical, hydraulic and mechanical characteristics of all the system components that are relevant to the purpose, including inertia, viscous and dry friction on the hydraulic piston, and a third order electromechanical model of the first and second stage dynamics of the servo-valve.

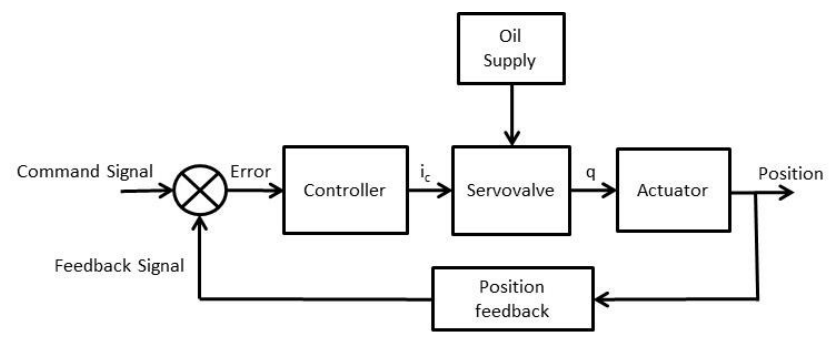

Fig. 6. Block diagram of the simulated test bench

The simulations shown in Figures 7 to 9 represent the dynamic response of the aforementioned actuator to a combination of position controls (Com), external loads (FR) and variations in the hydraulic supply pressure (PSR), for the different models of the second stage sliding spool valve. The sequence of input has been defined to appropriately highlight the performance of the proposed fluid-dynamic models and their effect on the dynamic behaviour of the simulation test bench $[6,18-21]$. The dynamic response of the full actuator in combination with the HF model of the spool valve is reported in Figure 7, to be taken as reference for the comparison with the simplified models.

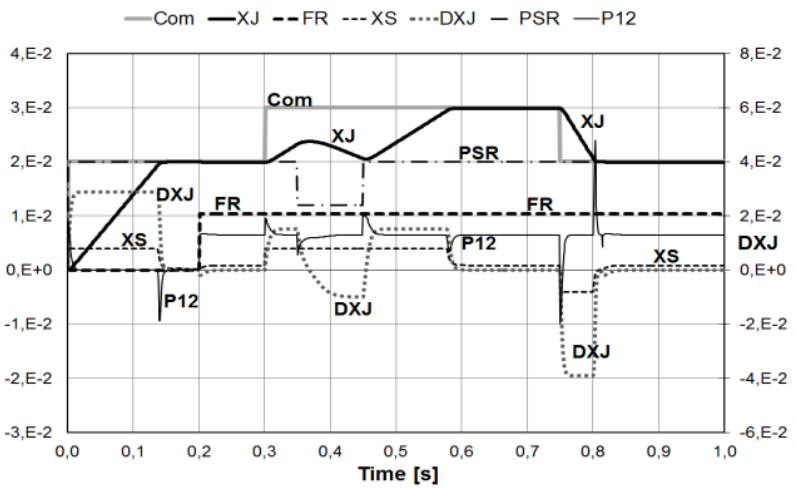

Fig. 7. Test bench simulation with the HF model; Com is the position command, $F_{R}$ the external load, $x_{S}$ the spool position, $d x_{J}$ the jack speed, $x_{J}$ the jack position, $P_{S R}$ the supply pressure, and $P_{12}$ the differential control pressure.

Figure 8 is the response of model C3. The model is able to replicate with satisfying accuracy the behaviour of the HD model for the unloaded actuation (from 0 to $0.2 \mathrm{~s}$ ) and the aiding load condition (at $0.75 \mathrm{~s}$ ), despite a slightly higher starting acceleration and a lower stopping deceleration, due to the inability to account for the water hammer effect. The simulation of the opposing load actuation (at $0.3 \mathrm{~s}$ ) underestimates the effect of the external load, and at the supply pressure drop $(0.35 \mathrm{~s})$ the back movement is overestimated. This behaviour can be ascribed to the lack of water-hammer effect, i.e. when the valve passageways are closed, the differential pressure should grow higher than $P_{S R}$ to resist an external load higher than the actuator stall force. Further, following a spool displacement, the jack acceleration has an almost constant value (as can be seen in the constant slope of $d x_{J}$ around $0.4 \mathrm{~s}$ ), rather than the asymptotic trend of the HF model; the reason lies in the simple but partially unsatisfying action of the $P_{12}$ saturation block. 


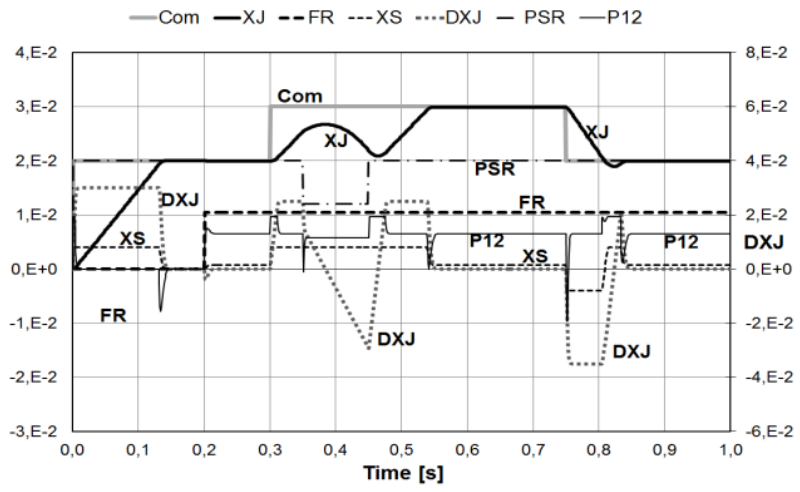

Fig. 8. Test bench simulation with the $\mathrm{C} 3$ model

Figure 9 is the response of model C5. The model behaviour is almost identical to the previous case, meaning that model $\mathrm{C} 5$ shares the same issues of $\mathrm{C} 3$, and the correction made to the leakage loop is negligible in terms of dynamic response of the whole system.

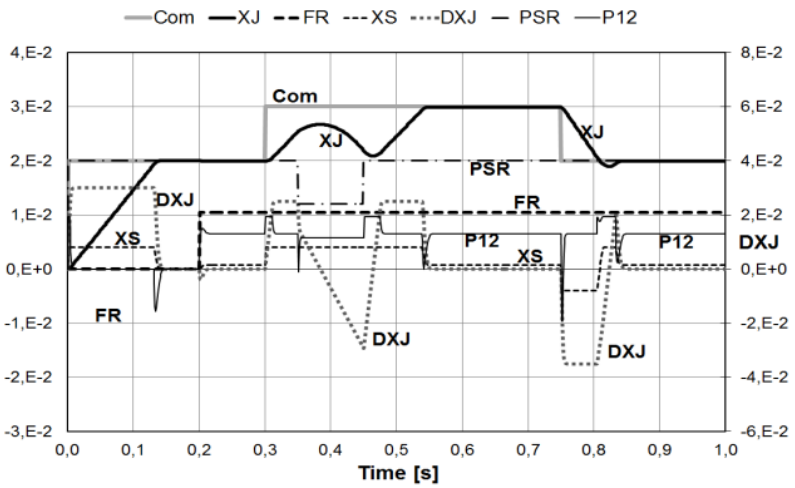

Fig. 9. Test bench simulation with the $\mathrm{C} 5$ model

\section{Conclusions}

The analysis of the considered servo-valve fluid-dynamic numerical models clearly highlights the few advantages and the important problems of the proposed simplified approaches. Transients (accelerations, decelerations), water-hammer and transitory pressure conditions are either overestimated or underestimated, depending on the particular operating condition; to date, a completely satisfying simplified servo-valve model, combining accuracy and low computational complexity, and featuring a dependency only on easily measurable parameters, is not available. In particular, the proposed C5 model is not capable of overcoming the issues of $\mathrm{C} 3$ model, from which it was derived.

In the authors' opinion, further studies are needed, in order to develop algorithms that are more efficient and improve the ability to perform acceptable simulations in all possible working conditions.

The authors wish to extend a heartfelt thanks to Professor Lorenzo Borello for his valuable teachings and for his support in the conception and development of this research. 


\section{References}

1. A. De Martin P. Chiavaroli, G. Evangelista, G. Jacazio, M. Sorli, Proceedings of the ASME 2018 International Mechanical Engineering Congress and Exposition (2018)

2. D. Henninger, A. Zopey, T. Ihde, C. Mehring, Int. J. Mechanical \& Mechatronics Eng. 11(8) (2017)

3. Q. Chen, B. Stoffel, ASME/JSME 2004 Pressure Vessels and Piping Conference (2004)

4. A. De Martin, A. Dellacasa, G. Jacazio, M. Sorli, BATH/ASME 2018 Symposium on Fluid Power and Motion Control (2018)

5. D.H. Kim, T.C. Tsao, J. of Dynamic systems Measurement and Control 122(1) (2000)

6. L. Borello, G. Villero, XI AIDAA National Congress (1991)

7. H. E. Merritt, Hydraulic control systems (Wiley, 1967).

8. T. J. Viersma, Analysis, synthesis and design of hydraulic servosystems and pipelines (Elsevier, 1980)

9. E. Urata, Bath workshop on power transmission and motion control, 269-282 (2000)

10. E. Urata, The Seventh Scandinavian Intl. Conf. on Fluid Power 1 51-66 (2001)

11. E. Urata, Proc. Fifth JFPS Intl. Symp. on Fluid Power 3 769-774 (2002)

12. E. Urata, The Eighth Scandinavian Intl. Conf. on Fluid Power 871-885 (2003)

13. E. Urata, $4^{\text {th }}$ IFK Workshop 71-82 (2004)

14. E. Urata, Int. J. of Fluid Power, 5(2) 35-42 (2004)

15. E. Urata, The Ninth Scandinavian Intl. Conf. on Fluid Power (2005)

16. W. Jia, C. Yin, $2^{\text {nd }}$ Intl. Conf. on Computer Engineering and Technology - IEEE, (2010)

17. X. Pan, G. Wang, Z. Lu, Energy Conversion and Management 52 3249-3256 (Elsevier, 2011)

18. P. Alimhillaj, L. Borello, M.D.L. Dalla Vedova, Int. J. of Mechanics and Control (JoMaC), 14(2) 39-49 (2013)

19. L. Borello, M.D.L. Dalla Vedova, Int. J. of Mechanics and Control (JoMaC), 8(2) 4352 (2007)

20. L. Borello, M.D.L. Dalla Vedova, XX AIDAA National Congress (2009)

21. P. Alimhillaj, M.D.L. Dalla Vedova, $3^{\text {rd }}$ European Conference on Electrical Engineering \& Computer Science (2018) 